\title{
Realization of Logical Circuits with Majority Logical Function as Symmetrical Function
}

\author{
J. Bokr, V. Jáněs
}

The paper deals with the "production" and design of symmetrical functions, particularly aimed at the design of circuits with majority elements, which lead to interesting solutions of logical structures. The solutions are presented in several examples, which show the applicability of the procedures to the design of FPGA morphology on chips.

Keywords: Shannon extension development, Hamming weight, derivation of Boolean function, symmetrical and majority function.

\section{Introduction}

Binary logical circuits designed with respect to Boolean symmetrical, particularly majority, output functions are certainly worth attention. The article, therefore, makes an evaluation both by controlling binary one-digit adders and by using functions interpreted by arithmetic polynomials. It also demonstrates how effectively the Shannon decomposition of the output functions can be used in designing a circuit with majority elements.

\section{Boolean function}

Let a Boolean function $f:\{0,1\}^{m} \rightarrow\{0,1\}:\left\langle x_{1}, x_{2}, \ldots, x_{m}\right\rangle$ and $y$ be given. If we denote the set $\left\{x_{i}\right\}_{i=1}^{m}$ of arguments $x_{1}$ by the symbol $X$, we can briefly write $f(X)$ instead of $f\left(x_{1}, x_{2}\right.$, $\left.\ldots, x_{m}\right)$. In addition, instead of $f\left(x_{1}, x_{2}, \ldots, x_{i-1}, \sigma_{i}, x_{i+1}, \ldots\right.$, $\left.x_{m}\right)$, in which $\sigma_{i} \in\{0,1\}$, let us simply write $f\left(x={ }_{i s}\right)$. Let $x^{\sigma}=x \sigma \vee \bar{x} \bar{\sigma}(\sigma \in\{0,1\})$; any Boolean function $f\left(x_{1}, x_{2}, \ldots\right.$, $x_{m}$ ) can be expressed, without loss of generality, by the Shannon extension development

$f(X)=$

$=\underset{\left\langle\sigma_{1}, \sigma_{2}, \ldots, \sigma_{n}\right\rangle}{\vee} x_{1}^{\sigma_{1}} x_{2}^{\sigma_{2}} \ldots x_{n}^{\sigma_{n}} f\left(\sigma_{1}, \sigma_{2}, \ldots, \sigma_{n}, x_{n+1}, \ldots, x_{m}\right)$

where $n \leq m$ esp.

$f(X)=\underset{\sigma_{i}}{\vee} x_{i}^{\sigma_{1}} f\left(x_{i}=\sigma_{1}\right)=\bar{x}_{i} f\left(x_{i}=0\right) \vee x_{i} f\left(x_{i}=1\right)$

the functions $f\left(\sigma_{1}, \sigma_{2}, \ldots, \sigma_{n}, x_{n+1}, \ldots, x_{m}\right)$ will be called remainder functions.

By the Hamming weight $w_{H} f(X)$ of the function $f(X)$ we understand the value of the arithmetic formula $w_{H} f(X)=\sum_{\left\langle\sigma_{1}, \sigma_{2}, \ldots, \sigma_{m}\right\rangle} f\left(\sigma_{1}, \sigma_{2}, \ldots, \sigma_{m}\right)$

The partial derivation $\frac{\partial f(X)}{\partial x_{i}}[1]$ of function $f(X)$ by the argument $x$ will be termed the Boolean function

$$
\begin{aligned}
\frac{\partial f(X)}{\partial x_{i}} & =f\left(x_{1}, x_{2}, \ldots, x_{i-1}, 0, x_{i+1}, \ldots, x_{m}\right) \oplus \\
& \oplus f\left(x_{1}, x_{2}, \ldots, x_{i-1}, 1, x_{i+1}, \ldots, x_{m}\right)
\end{aligned}
$$

defining the conditions under which $f(X)$ changes its value while the value of the argument $x$ is changed.

For example, for $y=\overline{x_{2}} \overline{x_{3}} \vee x_{1} x_{2} x_{3}$, when $w_{H} \frac{\partial y}{\partial x_{i}}=w_{H}\left(\overline{x_{2}} \overline{x_{3}} \vee\left(x_{2} \equiv x_{3}\right)\right)=w_{H}\left(x_{2} x_{3}\right)=1$

the function $y$ changes its value while the value of the argument $x_{1}$ is changed under one condition for $w_{H} \frac{\partial y}{\partial x_{i}}=1$, which is: $x_{2}=x_{3}=1$.

\section{Boolean formulae and arithmetic polynomials}

$\underline{\text { Let us have }}$

$$
f\left(x_{1}, x_{2}, \ldots, x_{m}\right)=\underset{i, k}{\vee} x_{i 1}^{\sigma_{i 1}} x_{i 2}^{\sigma_{i 2}} \ldots x_{i k}^{\sigma i k}
$$

where $\boldsymbol{k}=1,2, \ldots, m$ and $\boldsymbol{i}=0,1, \ldots, 2^{m}-1$, the function $f(X)$ is represented by a normal disjunctive formula $(n d f f(X))$. If there holds

$\left(\begin{array}{cccc}x_{i 1}^{\sigma} & x_{i 2}^{\sigma} & \ldots & x_{i k}^{\sigma} \sigma_{i k}\end{array}\right)\left(\begin{array}{cccc}x_{j 1}^{\sigma_{j 1}} & x_{j 2}^{\sigma} & \ldots & x_{j l}^{\sigma_{j l}}\end{array}\right)=0$,

where $k=1,2, \ldots, \boldsymbol{m}$ and $j=0,1, \ldots, 2^{m}-1$, the conjuncts presented are termed orthogonal, i.e., all conjuncts of a complete normal disjunctive formula of the symmetrical Boolean function (see Paragraph 4) are mutually orthogonal. If all conjuncts $n d f f(X)$ are mutually orthogonal, we can also write

$$
f\left(x_{1}, x_{2}, \ldots, x_{m}\right)=\bigoplus_{i, k} x_{i 1}^{\sigma} x_{i 2}^{\sigma} x_{i 2}^{\sigma} \ldots x_{i k}^{\sigma i k} .
$$

Note that the function $f(X)$ can also be conveniently expressed by the Boolean (Zegalkin) polynomial [4].

Since, as can easily be confirmed, the following equality holds:

$$
\begin{aligned}
& x \vee y=x+y-x y \\
& \text { (probability addition) } \\
& x y=x y \\
& \bar{x}=1-x
\end{aligned}
$$

and also ( $x$ and $\bar{x} y$ are orthogonal)

$$
x \vee y=x \vee \bar{x} y=x+(1-x) y,
$$


each $n d f f(X)$ can be expressed by an arithmetic polynomial

$f\left(x_{1}, x_{2}, \ldots, x_{m}\right)=a_{0}+a_{1} x_{1}+a_{2} x_{2}+\ldots+a_{m} x_{m}+a_{m+1} x_{1} x_{2}$

$+a_{m+2} x_{1} x_{2}+\ldots+a_{2 m-1} x_{1} x_{m}+\ldots+a_{2 m-1} x_{1} x_{2} \ldots x_{m}$

$=A\left(x_{1}, x_{2}, \ldots, x_{m}\right)$

where $a_{i} \in N\left(i=0,1, \ldots, 2^{m}-1\right)$, this can be done either by applying the equality $x \vee y=x+y-x y$, or orthogonalizing all conjuncts $n d f f(X)$ and applying the absorption $x \vee \bar{x} y=$ $=x+(1-x) y$. Note that if the Boolean function $f(X)$ is expressed by the arithmetic polynomial $A(X)$, then

$$
f(X)=\operatorname{sign} A(X) .
$$

Example 1.: Let $n d f f\left(x_{1}, x_{2}, x_{3}\right)=\left(x_{1} \vee x_{2}\right) . x_{3} \vee x_{1} x_{2}$ be given. Express the given formula by means of the arithmetic polynomial $A\left(x_{1}, x_{2}, x_{3}\right)$, which is $x x=x^{2}=x$ :

$$
\begin{aligned}
& \left(x_{1} \vee x_{2}\right) x_{3} \vee x_{1} x_{2}=x_{1} x_{2}+\left(x_{1} \vee x_{2}\right)\left(x_{3}-x_{1} x_{2} x_{3}\right)= \\
& =x_{1} x_{2}+\left(x_{1}+x_{2}-x_{1} x_{2}\right)\left(x_{3}-x_{1} x_{2} x_{3}\right)= \\
& =x_{1} x_{2}+x_{1} x_{3}+x_{2} x_{3}-2 x_{1} x_{2} x_{3}
\end{aligned}
$$

$$
\begin{aligned}
& \left(x_{1} \vee x_{2}\right) x_{3} \vee x_{1} x_{2}=x_{1} x_{2} \vee \overline{x_{1} x_{2}}\left(x_{1} \vee \overline{x_{1}} x_{2}\right) x_{3}= \\
& =x_{1} x_{2} \vee\left(\overline{x_{1}} \vee \overline{x_{2}}\right)\left(x_{1} \vee \overline{x_{1}} x_{2}\right) x_{3}=x_{1} x_{2} \vee\left(x_{1} \overline{x_{2}} \vee \overline{x_{1}} x_{2}\right) x_{3}= \\
& =x_{1} x_{2}+\left(x_{1}\left(1-x_{2}\right)+\left(1-x_{1}\right) x_{2}\right) x_{3}= \\
& =x_{1} x_{2}+x_{1} x_{3}+x_{2} x_{3}-2 x_{1} x_{2} x_{3}
\end{aligned}
$$

\section{Symmetrical Boolean function}

Let the bijection $X \leftrightarrow X$ :

$\left\langle x_{1}, x_{2}, \ldots, x_{m}\right\rangle \leftrightarrow\left\langle x_{i 1}, x_{i 2}, \ldots, x_{i m}\right\rangle$ be a set of all permutations of arguments from $X$; the function $f(X)$ is called symmetrical if $f\left(x_{1}, x_{2}, \ldots, x_{m}\right)=f\left(x_{i 1}, x_{i 2}, \ldots, x_{i m}\right)$; i.e. $0, x \oplus y$, $x y \vee x z \vee y z$ is a symmetrical function. Let $\left\{P_{j}\right\}_{j=1}^{k}$ be a set of integers $P_{j}$ (called operational or characteristic numbers) such that $0 \leq P_{j} \leq m$. It can be demonstrated [2] that $f(X)$ is symmetrical just if $f\left(\sigma_{1}, \sigma_{2}, \ldots, \sigma_{m}\right)=1$ for $w_{H}\left(\sigma_{1}, \sigma_{2}, \ldots, \sigma_{m}\right)=P_{j}$. The symmetrical function with characteristic numbers $P_{j}$ will be denoted $S_{\left\{P_{j}\right\}_{j=1}^{m}}^{k}$;

obviously, $S_{\varnothing}^{m}=0$ and $S_{\{0,1, \ldots, m\}}^{m}=1$.

For example: $S_{\{1\}}^{2}=x \oplus y, S_{\{2,3\}}^{3}=x y \vee x z \vee y z$.

The symmetrical function $S_{\{P\}}^{m}$ is elementary; for the length $\left|\operatorname{cndf} S_{\{P\}}^{m}\right|$ of the completely normal disjunctive formula $S_{\{P\}}^{m}$ - there holds $\left|\operatorname{Cndf} S_{\{P\}}^{m}\right|=m\left(\begin{array}{c}m \\ P\end{array}\right)$, since

$\operatorname{Cndf} S_{\{P\}}^{m}=\underset{\substack{\left\langle\sigma_{1}, \sigma_{2}, \ldots, \sigma_{m}\right\rangle \\ w_{H}\left(\sigma_{1} \sigma_{2} \ldots \sigma_{n}\right)=P}}{\vee} x_{1}^{\sigma_{1}} x_{2}^{\sigma_{2}} \ldots x_{m}^{\sigma_{m}}$.

There also holds [2]
$\left|\underset{\{Q\} \in 2^{\{0,1, \ldots, m\}}}{\cup}\left\{S_{\{Q\}}^{m}\right\}\right|=\sum_{i=0}^{m+1}\left(\begin{array}{c}m+1 \\ i\end{array}\right)=2^{m+1}$ as well as

$S_{\left\{P_{i}\right\}}^{m} \cdot S_{\left\{P_{j}\right\}}^{m}=0$ for $P_{i} \neq P_{j}$. Any symmetrical function $S_{\left\{P_{j}\right\}_{j=1}^{k}}^{m}$ can be written in the form of $\operatorname{cndf} S_{\left\{P_{j}\right\}_{j=1}^{k}}^{k}$ : $\left.S^{m} P_{j}\right\}_{j=1}^{k}=\underset{\substack{\left\langle\sigma_{1}, \sigma_{2}, \ldots, \sigma_{m}\right\rangle \\ w_{H}\left(\sigma_{1} \sigma_{2} \ldots \sigma_{m}\right)=P_{1}, P_{2}, \ldots, P_{k}}}{\vee} x_{1}^{\sigma_{1}} x_{2}^{\sigma_{2} \ldots x_{m}^{\sigma_{m}}}$ since [2] $S_{\left\{P_{j}\right\}_{j=1}^{k}}^{k}=\underset{j=1}{k} S_{\left\{P_{j}\right\}}^{m}$. We can also write

$$
S_{\left\{P_{j}\right\}_{j=1}^{k}}^{k}=\underset{j=0}{\mathrm{v}} \tau_{i} S_{\{i\}}^{m}
$$

where $\tau_{i}=\left\{\begin{array}{lll}1 & \text { for } & i=P_{j} \\ 0 & \text { for } & i \neq P_{j}\end{array}\right.$.

Denote the elementary symmetrical Boolean functions the representation of which in the form of normal disjunctive formulae $(n d f)$ does not contain negated variables with the symbol $S_{\{n\}}^{m}(n=0,1, \ldots, m)$.

For example

$S_{\{0\}}^{m}=1$,

$S_{\{1\}}^{m}=x_{1} x_{2} \ldots x_{m}, \ldots$,

$S_{\{m-1\}}^{m}=x_{1} x_{2} \vee x_{1} x_{3} \vee \ldots \vee x_{m-1} x_{m}$,

$S_{\{m\}}^{m}=x_{1} \vee x_{2} \vee \ldots \vee x_{m}$.

Every function $S_{\{n\}}^{m}$ in which $n \neq m\left(S_{\{n\}}^{m}=x_{1} x_{2} \ldots x_{m}\right)$, can be expressed by the composition

$$
S_{\{n\}}^{m}=S_{\{n\}}^{m} \overline{S_{\{m-n\}}^{m}} .
$$

For example:

$S_{\{2\}}^{3}=S_{\{2\}}^{3} \cdot \bar{S}_{\{1\}}^{3}=\left(x_{1} x_{2} \vee x_{1} x_{3} \vee x_{2} x_{3}\right) \overline{x_{1} x_{2} x_{3}}=$

$=x_{1} x_{2} \overline{x_{3}} \vee x_{1} \overline{x_{2}} x_{3} \vee \overline{x_{1}} x_{2} x_{3}, \quad$ resp.

$S_{\{2\}}^{3}=S_{\{1\}}^{3} \cdot \bar{S}_{\{2\}}^{3}=x_{1} x_{2} x_{3} \overline{x_{1} x_{2} \vee x_{1} x_{3} \vee x_{2} x_{3}}=$

$=x_{1} x_{2} x_{3}\left(\overline{x_{1}} \overline{x_{2}} \vee \overline{x_{1}} \overline{x_{3}} \vee \overline{x_{2}} \overline{x_{3}}\right)=x_{1} \overline{x_{2}} \overline{x_{3}} \vee \overline{x_{1}} x_{2} \overline{x_{3}} \vee \overline{x_{1}} \overline{x_{2}} x_{3}$.

Symmetrical functions are discussed in greater detail, e.g., in $[2,3,4]$.The majority function $M a j_{\{M\}}^{m}$ refers to the symmetrical function

$$
\underset{i=0}{\mathrm{v}} S_{\{M+i\}}^{m}=S_{\{M, M+1, \ldots, m\}}^{m} .
$$

For the three-variable majority function

$$
\operatorname{Maj}_{\{2\}}^{3}=x_{1} x_{2} \vee x_{1} x_{3} \vee x_{2} x_{3}
$$

an infix notation $x_{1} \# x_{1} \# \# x_{3}$ can be used. There obviously holds $x_{1} \# x_{1} \# x_{3}=x_{1}$ and $x_{1} \# \overline{x_{1}} \# x_{3}=x_{3}$.

\section{Numerical representation of symmetrical functions}

We might construct a minimal normal disjunctive formula to a given symmetrical function [4] or decompose the given 
a)

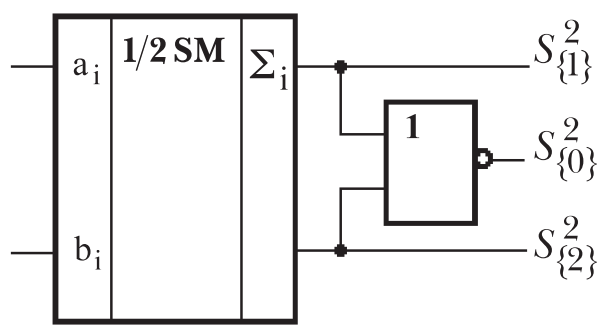

b)

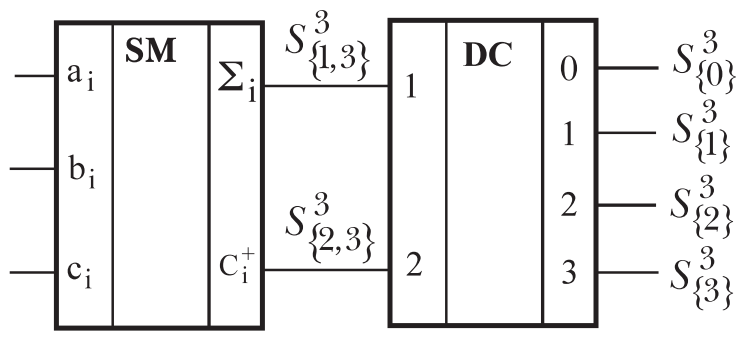

Fig. 1: a) Half-adder, b) Adder

function $\left(S_{\{n\}}^{m}=S_{\{n\}}^{m} \overline{S_{\{m-n\}}^{m}}\right)$ and design, according to the constructed formulae, a structural model of the given function in one of the structurally complete systems of statistical elements [5].

Further, consider a one-digit binary half-adder or an ad$\operatorname{der}$ (Fig. 1) with which $a_{i}, b_{i}$ are binary augmenters, $\Sigma_{i}$ is the sum in $i$ position, and $C_{i}^{-}$and $C_{i}^{+}$denote the transfer from the position $i-1$ to the position $i+1$, respectively. The half-adder can be modeled by a system of output functions $\Sigma_{i} a_{i} \oplus b_{i}=S_{\{1\}}^{2}$ and $C_{i}^{+}=a_{i} b_{i}=S_{\{2\}}^{2}$; by analogy for the adder we obtain

$\Sigma_{i}=a_{i} \oplus b_{i} \oplus C_{i}^{-}=S_{\{1,3\}}^{3}$ and

$C_{i}^{+}=\overline{a_{i}} b_{i} C_{i}^{-} \vee a_{i} \overline{b_{i}} C_{i}^{-} \vee a_{i} b_{i} \overline{C_{i}^{-}} \vee a_{i} b_{i} C_{i}^{-}=S_{\{2,3\}}^{3}$.

It is therefore sufficient to provide the half-adder with an inverse disjunctor (Fig.1a) and the adder with a decoder (Fig. 1b) and we obtain the products $S_{\{0\}}^{2}, S_{\{1\}}^{2}, S_{\{2\}}^{2}$, since

$$
\overline{S_{\{1\}}^{2}} \overline{S_{\{2\}}^{2}}=S_{\{0,2\}}^{2} S_{\{0,1\}}^{2}=S_{\{0\}}^{2}
$$

and $S_{\{0\}}^{3}, S_{\{1\}}^{3}, S_{\{2\}}^{3}, S_{\{3\}}^{3}$, since

$$
\begin{aligned}
& \overline{S_{\{1,3\}}^{3}} \cdot \overline{S_{\{2,3\}}^{3}}=S_{\{0,2\}}^{3} \cdot S_{\{0,1\}}^{3}=S_{\{0\}}^{3}, \\
& S_{\{1,3\}}^{3} \cdot \overline{S_{\{2,3\}}^{3}}=S_{\{1,3\}}^{3} \cdot S_{\{0,1\}}^{3}=S_{\{1\}}^{3}, \\
& \overline{S_{\{1,3\}}^{3}} \cdot S_{\{2,3\}}^{3}=S_{\{0,2\}}^{3} \cdot S_{\{2,3\}}^{3}=S_{\{2\}}^{3}, \\
& \overline{S_{\{1,3\}}^{3}} \cdot \overline{S_{\{2,3\}}^{3}}=S_{\{3\}}^{3} .
\end{aligned}
$$

Example 2.: Design a structural model with adders or half-adders modeled with a system of output symmetrical functions $S$ - Fig. 2. Indeed,

$\Sigma_{1}=x_{1} \oplus x_{2} \oplus x_{3}=\left(x_{1} \oplus x_{2}\right) \overline{x_{3}} \vee\left(x_{1} \equiv x_{2}\right) x_{3}=S_{\{1,3\}}^{3}$,

$C_{1}=S_{\{2,3\}}^{3}$,

$\Sigma_{2}=x_{4} \oplus S_{\{1,3\}}^{3}=\overline{x_{4}} S_{\{1,3\}}^{3} \vee x_{4} \overline{S_{\{1,3\}}^{3}}=$

$=S_{\{1,3\}}^{4} \vee x_{4} S_{\{0,2\}}^{3}=S_{\{1,3\}}^{4} \vee S_{\{1,3\}}^{4}=S_{\{1,3\}}^{4}$,

$C_{2}=x_{4} S_{\{1,3\}}^{3}=S_{\{2,4\}}^{4}$,
$\Sigma_{3}=$

$=S_{\{2,4\}}^{4} \oplus S_{\{2,3\}}^{3}\left(\overline{x_{4}} \oplus x_{4}\right)=$

$=\left(S_{\{0,1,3\}}^{4} S_{\{1,3\}}^{3} \vee S_{\{2,4\}}^{4} S_{\{0,1\}}^{3}\right)\left(\overline{x_{4}} \vee x_{4}\right)=$

$=S_{\{0,1,3\}}^{4} S_{\{2,3\}}^{4} \vee S_{\{2,4\}}^{4} S_{\{0,1\}}^{4} \vee S_{\{0,1,3\}}^{4} S_{\{3,4\}}^{4} \vee S_{\{2,4\}}^{4} S_{\{1,2\}}^{4}=$

$=S_{\{3\}}^{4} \vee S_{\{2\}}^{4}=S_{\{2,3\}}^{4}$,

$C_{3}=S_{\{2,4\}}^{4} S_{\{2,3\}}^{3}\left(\overline{x_{4}} \vee x_{4}\right)=S_{\{2,4\}}^{4} S_{\{2,3\}}^{4} \vee S_{\{2,4\}}^{4} S_{\{3,4\}}^{4}=$

$=S_{\{2\}}^{4} \vee S_{\{4\}}^{4}=S_{\{2,4\}}^{4}$.

It is easy to obtain

$S_{\{1,3\}}^{4}=S_{\{0,2,4\}}^{4}, \overline{S_{\{2,3\}}^{4}}=S_{\{0,1,4\}}^{4}$ and $\overline{S_{\{2,4\}}^{4}}=S_{\{0,1,3\}}^{4}$; hence for the decoder:

$S_{\{0\}}^{4}=S_{\{1,3\}}^{4} S_{\{2,4\}}^{4}, S_{\{2\}}^{4}=S_{\{2,3\}}^{4} S_{\{2,4\}}^{4}, S_{\{3\}}^{4}=S_{\{1,3\}}^{4} S_{\{2,3\}}^{4}$,

$S_{\{1\}}^{4}=S_{\{0,1,3\}}^{4} S_{\{0,1,4\}}^{4} S_{\{1,3\}}^{4}=\overline{S_{\{2,4\}}^{4}} \overline{S_{\{2,3\}}^{4}} S_{\{1,3\}}^{4}$,

$S_{\{4\}}^{4}=S_{\{0,1,4\}}^{4} S_{\{0,2,4\}}^{4} S_{\{2,4\}}^{4}=\overline{S_{\{2,3\}}^{4}} S_{\{1,3\}}^{4} S_{\{2,4\}}^{4}$.

If the Boolean function $f(X)$ is symmetrical, it can be suitably expressed by an arithmetic polynomial in the form

$f\left(x_{1}, x_{2}, \ldots, x_{m}\right)=b_{0}+b_{1}\left(x_{1}+x_{2}+\ldots+x_{m}\right)+$

$+b_{2}\left(x_{1} x_{2}+x_{2} x_{3}+\ldots+x_{m-1} x_{m}\right)+b_{3}\left(x_{1} x_{2} x_{3}+x_{1} x_{2} x_{4}+\right.$

$\left.+\ldots+x_{m-2} x_{m-1} x_{m}\right)+\ldots+b_{m+1} x_{1} x_{2}+\ldots+x_{m}=$

$=b_{0}+b_{1} X^{1}+b_{2} X^{2}+\ldots+b_{m} X^{m}=\sum_{i=0}^{m} b_{i} X^{i}$

for example for

$S_{\{1,2\}}^{3}=\overline{x_{1}} x_{2} \vee x_{1} \overline{x_{2}} \vee x_{1} x_{2}=\left(1-x_{1}\right) x_{2}+x_{1} x_{2}=x_{1}+x_{2}-x_{1} x_{2}$

we obtain $b_{0}=0, b_{1}=1, b_{2}=-1$ and

$X^{0}=1, X^{1}=x_{1}+x_{2}, X^{2}=x_{1} x_{2}$.

The parametric notation cndf $S_{\left\{P_{j}\right\}_{j=1}^{m}}^{k}$ being

$S_{\left\{P_{j}\right\}_{j=1}^{k}}^{m}=\underset{P_{j}}{\underset{\vee}{*}} X^{P_{j}} \bar{X}^{\left(m-P_{j}\right)}=\sum_{P_{j}}\left[m /\left(\begin{array}{c}m \\ P_{j}\end{array}\right)\right] \mathrm{X}^{\mathrm{P}_{\mathrm{j}}}(1-X)^{m-P_{j}}$ 


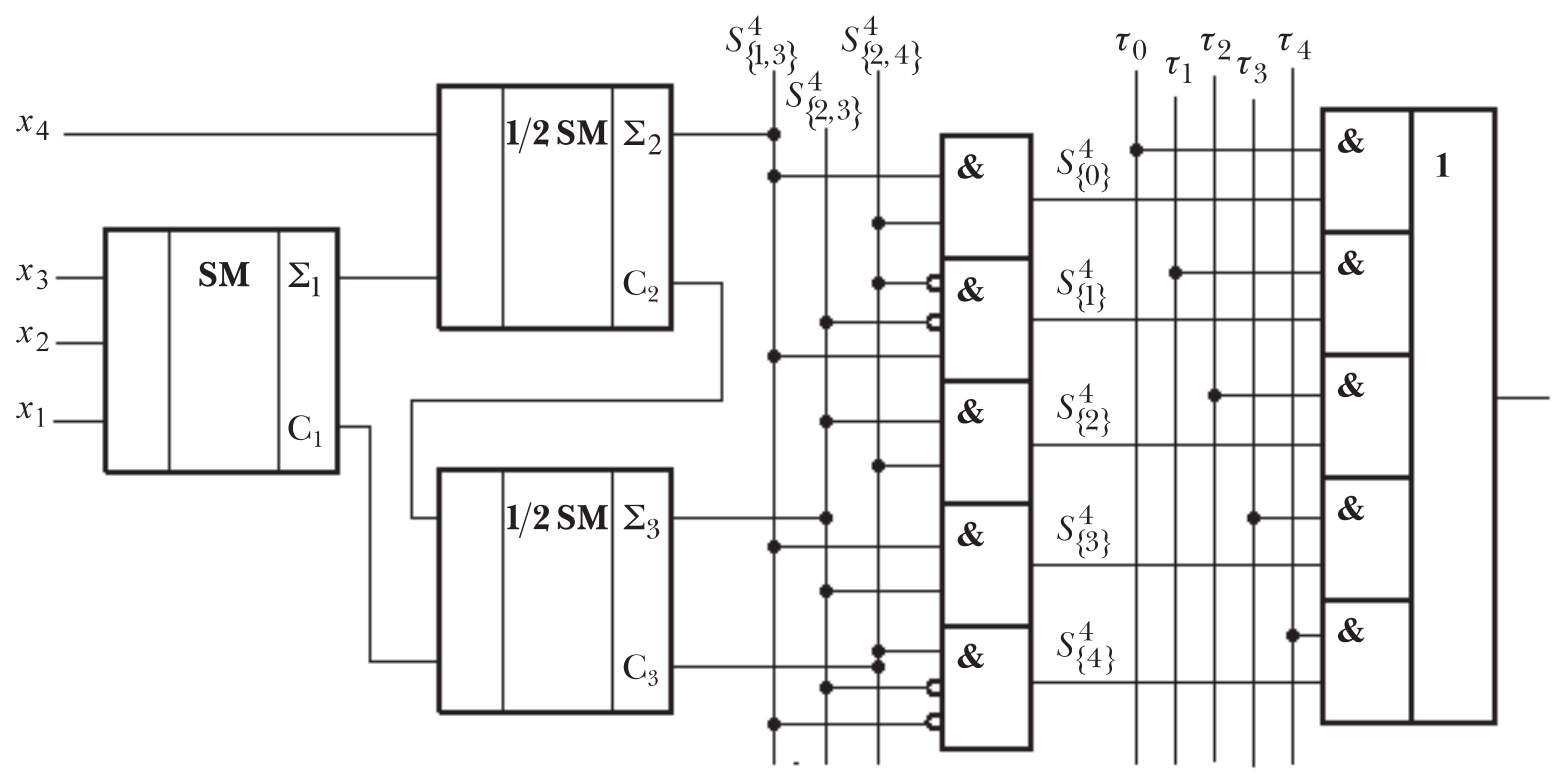

Fig. 2: Production of symmetrical functions $S_{\{i\}}^{4}(i=0,1,2,3,4)$ from Example 2

Hence

$$
\sum_{i=0}^{m} b_{i} X^{i}=\sum_{P_{j}}\left[m /\left(\begin{array}{c}
m \\
P_{j}
\end{array}\right)\right] \mathrm{X}^{\mathrm{P}_{\mathrm{j}}}(1-X)^{m-P_{j}}
$$

we can easily (!) determine the values of coefficients $b$, provided the structure of polynomials $X^{i}$ is known.

Example 3.: Construct an arithmetic polynomial of the symmetrical function $S_{\{1,2\}}^{3}$. Since

$$
\begin{aligned}
S_{\{1,2\}}^{3} & =\underset{\substack{\left\langle\sigma_{1}, \sigma_{2}, \sigma_{3}\right\rangle \\
w_{H}\left(\sigma_{1} \sigma_{2} \sigma_{3}\right)}}{\vee x_{1}^{\sigma_{1}} x_{2}^{\sigma_{2}} x_{3}^{\sigma_{3}}=\overline{x_{1}} \overline{x_{2}} x_{3} \vee \overline{x_{1}} x_{2} \overline{x_{3}} \vee} \\
& \vee x_{1} \overline{x_{2}} \overline{x_{3}} \vee \overline{x_{1}} x_{2} x_{3} \vee x_{1} \overline{x_{2}} x_{3} \vee x_{1} x_{2} \overline{x_{3}}= \\
& =\sum_{j=1,2}\left[3 /\left(\begin{array}{l}
3 \\
j
\end{array}\right)\right] X^{j}(1-X)^{3-j}= \\
& =\left[3 /\left(\begin{array}{l}
3 \\
1
\end{array}\right)\right] X(1-X)^{2}+\left[3 /\left(\begin{array}{l}
3 \\
2
\end{array}\right)\right] X^{2}(1-X)= \\
& =X-X^{2}=b_{0}+b_{1} X^{1}+b_{2} X^{2}+b_{3} X^{3} .
\end{aligned}
$$

Hence $b_{0}=b_{3}=0, b_{2}=1, b_{3}=-1$ provided that $X^{1}=x_{1}+x_{2}+x_{3}$ and $X^{2}=x_{1} x_{2}+x_{1} x_{3}+x_{2} x_{3}$

because

$S_{\{1,2\}}^{3}=\left(1-x_{1}\right) x_{2} x_{3}+x_{1}\left(1-x_{2}\right) x_{3}+x_{1} x_{2}\left(1-x_{3}\right)+$ $+\left(1-x_{1}\right)\left(1-x_{2}\right) x_{3}+\left(1-x_{1}\right) x_{2}\left(1-x_{3}\right)+x_{1}\left(1-x_{2}\right)\left(1-x_{3}\right)=$. $=\left(x_{1}+x_{2}+x_{3}\right)-2\left(x_{1} x_{2}+x_{1} x_{3}+x_{2} x_{3}\right)+3 x_{1} x_{2} x_{3}+$ $+\left(x_{1}+x_{2}+x_{3}\right)+\left(x_{1} x_{2}+x_{1} x_{3}+x_{2} x_{3}\right)-3 x_{1} x_{2} x_{3}$.

\section{Circuits with majority elements}

Let us limit ourselves to majority elements modeled with the function $M a j_{\{2\}}^{3}$.

Since, as can be easily confirmed, there holds

$$
\begin{aligned}
& x \vee y=x \# y \# 1, \\
& x y=x \# y \# 0,
\end{aligned}
$$

the $n d f$ of the given function $f(X)$ can be rewritten according to the above quoted equities and we can design the respective static structural model.

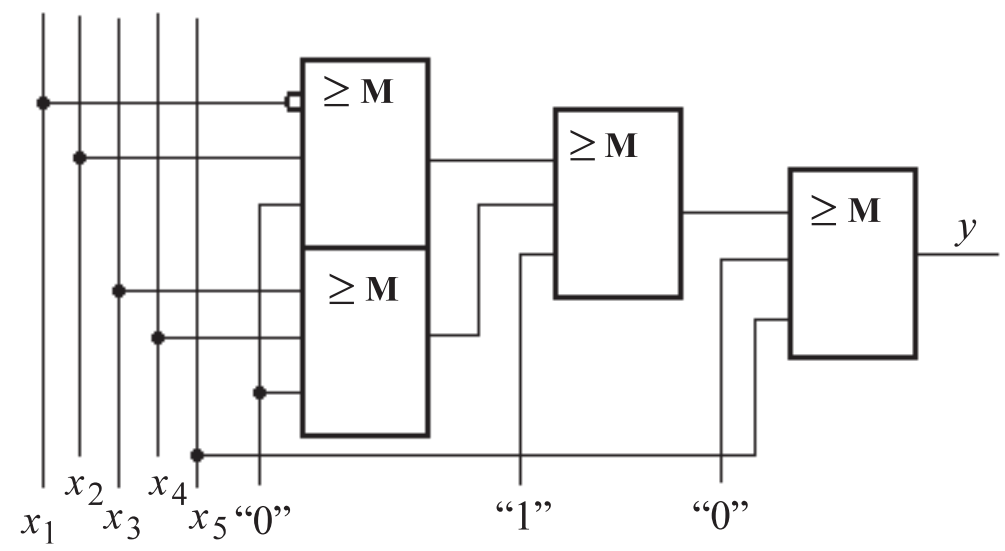

Fig. 3: Structural model from Example 4 
Example 4.: Construct a structural model given by the output function

$$
y=\left(\overline{x_{1}} x_{2} \vee x_{3} x_{4}\right) x_{5}
$$

hence

$$
y=\left(\left(\left(\overline{x_{1}} \# x_{2} \# 0\right) \#\left(x_{3} \# x_{4} \# 0\right)\right) \# 1\right) \# x_{5} \# 0 \text {, }
$$

and thus Fig. 3 .

It is also helpful to use the Shannon extension development of the given Boolean output function $y=f(X)$

$y=\overline{x_{i}} f\left(x_{i}=0\right) \vee x_{i} f\left(x_{i}=1\right)=$

$=\left(\overline{x_{i}} \# f\left(x_{i}=0\right) \# 0\right) \#\left(x_{i} \# f\left(x_{i}=1\right) \# 0\right) \# 1$,

and it remains only to decide according to which argument to start and according to which arguments to continue the repeated application of the development. We, therefore, heuristically develop $f(X)$, first according to the arguments whose change of values leads to the change of values $f(X)$ under the highest number of conditions, i.e., at the highest.

Hamming weights pertaining to the derivation of the function $f(X)$ according to the respective arguments.

Example 5.: Design a structural model given by the output function

$y=x_{1} \overline{x_{2}} x_{3} \vee \overline{x_{1}} \overline{x_{3}} x_{4} \vee x_{1} x_{3} \overline{x_{5}} \vee x_{1} x_{2} x_{4} \vee \overline{x_{2}} x_{3} x_{5} \vee \overline{x_{3}} \overline{x_{4}} x_{5}$ with majority elements. According to the map of the given output function, the $n d f$ of the remainder functions of its Shannon extension development can easily be constructed (Fig. 4.).

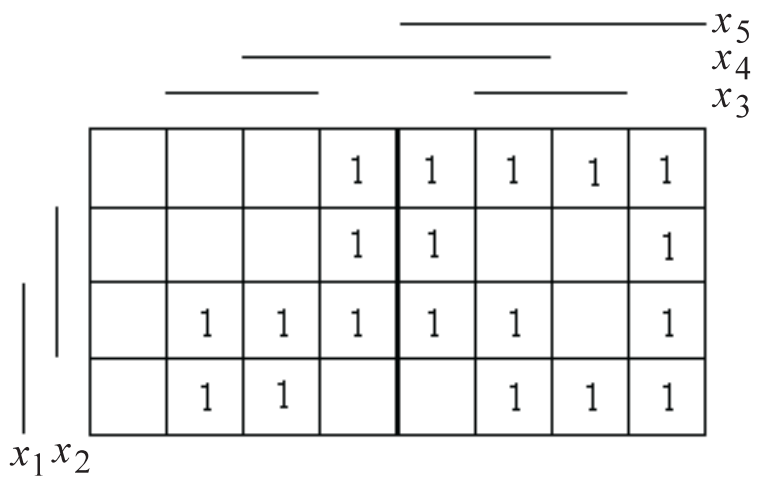

Fig. 4: The Karnaugh map of the output function from Example 5

Hence

$$
\begin{aligned}
& w_{H} \frac{\partial y}{\partial x_{1}}=w_{H}\left(y\left(x_{1}=0\right) \oplus y\left(x_{1}=1\right)\right)= \\
&=w_{H}\left(\left(\overline{x_{2}} x_{5} \vee \overline{x_{3}} x_{4} \vee \overline{x_{3}} x_{5}\right) \oplus\right. \\
&\left.\oplus\left(\overline{x_{2}} x_{3} x_{5} \vee x_{2} x_{4} \vee \overline{x_{3}} \overline{x_{4}} x_{5} \vee x_{3} \overline{x_{5}}\right)\right)=7
\end{aligned}
$$

When stating the formula which expresses the derivation of the function we will preferably use a map, to each field of which we will write the value in the form of a fraction:

$$
y\left(x_{i}=0\right) / y\left(x_{i}=1\right)
$$

the resulting value of the remainder function formula as well as the weight of its derivation is evident (Fig. 5). There also holds

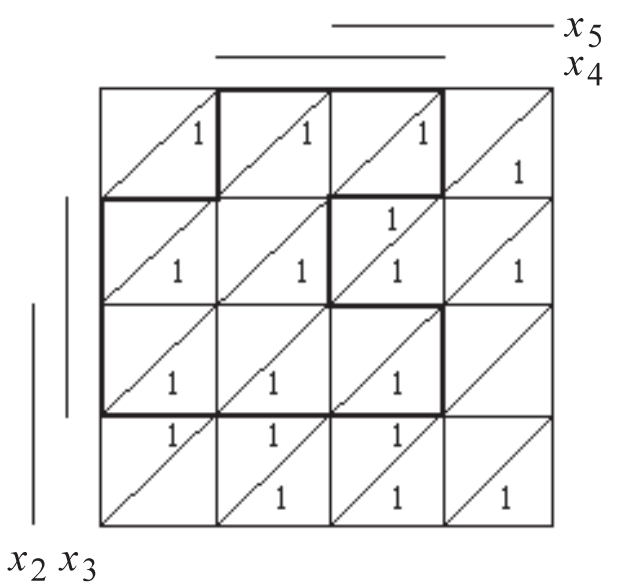

Fig. 5: Recording of remainder functions $\frac{\partial y}{\partial x_{1}}$ from Example 5

$w_{H} \frac{\partial y}{\partial x_{2}}=w_{H}\left(y\left(x_{2}=0\right) \oplus y\left(x_{2}=1\right)\right)=$ $=w_{H}\left(\left(\overline{x_{1}} \overline{x_{3}} x_{4} \vee x_{1} x_{3} \vee x_{3} x_{5} \vee \overline{x_{4}} x_{5}\right) \oplus\right.$

$\left.\oplus\left(x_{1} x_{4} \vee x_{1} x_{3} \overline{x_{5}} \vee \overline{x_{3}} x_{4} \vee \overline{x_{3}} x_{5}\right)\right)=5$,

$w_{H} \frac{\partial y}{\partial x_{3}}=w_{H}\left(y\left(x_{3}=0\right) \oplus y\left(x_{3}=1\right)\right)=$

$=w_{H}\left(\left(\overline{x_{1}} x_{4} \vee x_{2} x_{4} \vee \overline{x_{4}} x_{5}\right) \oplus\right.$

$\left.\oplus\left(x_{1} \overline{x_{5}} \vee x_{1} x_{4} x_{5} \vee \overline{x_{2}} x_{5}\right)\right)=8$,

$w_{H} \frac{\partial y}{\partial x_{4}}=w_{H}\left(y\left(x_{4}=0\right) \oplus y\left(x_{4}=1\right)\right)=$

$=w_{H}\left(\left(x_{1} \overline{x_{2}} x_{5} \vee x_{1} x_{3} \overline{x_{5}} \vee x_{1} \overline{x_{4}} x_{5}\right) \oplus\right.$

$\left.\oplus\left(\overline{x_{1}} \overline{x_{3}} \vee x_{1} x_{3} \vee x_{2} \overline{x_{3}}\right)\right)=5$,

$w_{H} \frac{\partial y}{\partial x_{5}}=w_{H}\left(y\left(x_{5}=0\right) \oplus y\left(x_{5}=1\right)\right)=$

$=w_{H}\left(\left(x_{1} x_{3} \vee \overline{x_{1}} \overline{x_{3}} x_{4} \vee x_{2} \overline{x_{3}} x_{4}\right) \oplus\right.$

$\left.\oplus\left(\overline{x_{1}} \overline{x_{3}} \vee \overline{x_{2}} x_{3} \vee x_{2} \overline{x_{3}} \vee \overline{x_{3}} \overline{x_{4}} \vee x_{1} x_{3} x_{4}\right)\right)=7$.

Since

$$
\max _{i}\left\{w_{H} \frac{\partial y}{\partial x_{i}}\right\}=w_{H} \frac{\partial y}{\partial x_{3}}=8,
$$

we write

$$
y=x_{3} y\left(x_{3}=0\right) \vee x_{3} y\left(x_{3}=1\right),
$$

where

$$
y\left(x_{3}=0\right)=\overline{x_{1}} x_{4} \vee x_{2} x_{4} \vee \overline{x_{4}} x_{5}
$$

and

$$
y\left(x_{3}=1\right)=x_{1} x_{4} \vee x_{1} \overline{x_{5}} \vee \overline{x_{2}} x_{5} \text { (Fig. 6). }
$$

And, further, there is

$$
\begin{aligned}
& w_{H} \frac{\partial y\left(x_{3}=0\right)}{\partial x_{1}}=w_{H}\left(y\left(x_{1}=0, x_{3}=0\right) \oplus y\left(x_{1}=1, x_{3}=0\right)\right)= \\
& =w_{H}\left(\left(x_{4} \vee x_{5}\right) \oplus\left(x_{2} x_{4} \vee \overline{x_{4}} x_{5}\right)\right)=2,
\end{aligned}
$$



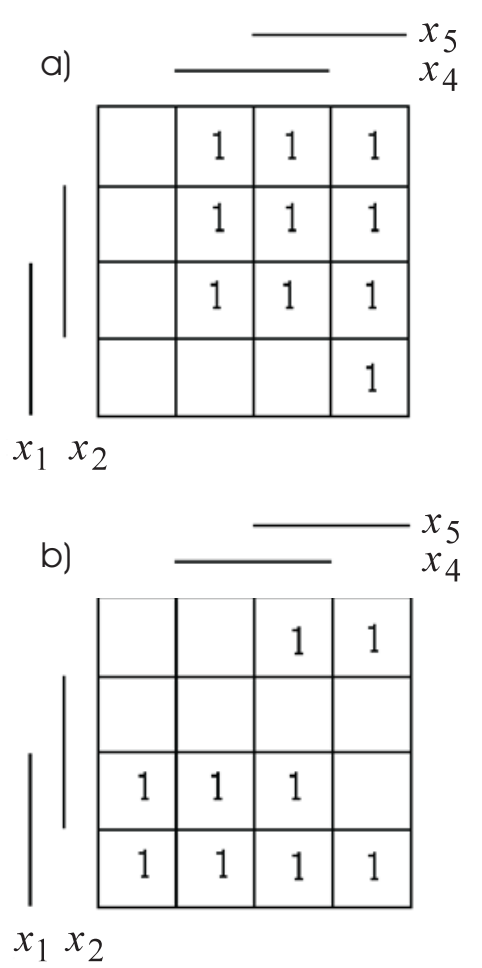

Fig. 6: Map entries of a) $y\left(x_{3}=0\right)$, b) $y\left(x_{3}=1\right)$ from Example 5

$$
\begin{aligned}
& w_{H} \frac{\partial y\left(x_{3}=0\right)}{\partial x_{2}}=w_{H}\left(y\left(x_{2}=0, x_{3}=0\right) \oplus y\left(x_{2}=1, x_{3}=0\right)\right)= \\
& =w_{H}\left(\left(\overline{x_{1}} x_{4} \vee \overline{x_{4}} x_{5}\right) \oplus\left(x_{4} \vee x_{5}\right)\right)=2, \\
& w_{H} \frac{\partial y\left(x_{3}=0\right)}{\partial x_{4}}=w_{H}\left(y\left(x_{3}=0, x_{4}=0\right) \oplus y\left(x_{3}=0, x_{4}=1\right)\right)= \\
& =w_{H}\left(x_{5} \oplus\left(\overline{x_{1}} \vee x_{2}\right)\right)=4,
\end{aligned}
$$

$$
\begin{aligned}
& w_{H} \frac{\partial y\left(x_{3}=0\right)}{\partial x_{5}}=w_{H}\left(y\left(x_{3}=0, x_{5}=0\right) \oplus y\left(x_{3}=0, x_{5}=1\right)\right)= \\
& =w_{H}\left(\left(\overline{x_{1}} x_{4} \vee x_{2} x_{4}\right) \oplus\left(\overline{x_{1}} \vee x_{2} \vee \overline{x_{4}}\right)\right)=4, \\
& w_{H} \frac{\partial y\left(x_{3}=1\right)}{\partial x_{1}}=w_{H}\left(y\left(x_{1}=0, x_{3}=1\right) \oplus y\left(x_{1}=1, x_{3}=1\right)\right)= \\
& =w_{H}\left(\overline{x_{2}} x_{5} \oplus\left(\overline{x_{2}} \vee x_{4} \vee \overline{x_{5}}\right)\right)=5, \\
& w_{H} \frac{\partial y\left(x_{3}=1\right)}{\partial x_{2}}=w_{H}\left(y\left(x_{2}=0, x_{3}=1\right) \oplus y\left(x_{2}=1, x_{3}=1\right)\right)= \\
& =w_{H}\left(\left(x_{1} \vee x_{5}\right) \oplus\left(x_{1} x_{4} \vee x_{1} \overline{x_{5}}\right)\right)=3, \\
& w_{H} \frac{\partial y\left(x_{3}=1\right)}{\partial x_{4}}=w_{H}\left(y\left(x_{3}=1, x_{4}=0\right) \oplus y\left(x_{3}=1, x_{4}=1\right)\right)= \\
& =w_{H}\left(\left(x_{1} \overline{x_{5}} \vee \overline{x_{2}} x_{5}\right) \oplus\left(x_{1} \vee \overline{x_{2}} x_{5}\right)\right)=1, \\
& w_{H} \frac{\partial y\left(x_{3}=1\right)}{\partial x_{5}}=w_{H}\left(y\left(x_{3}=1, x_{5}=0\right) \oplus y\left(x_{3}=1, x_{5}=1\right)\right)= \\
& =w_{H}\left(x_{1} \oplus\left(\overline{x_{2}} \vee x_{1} x_{4}\right)\right)=3 .
\end{aligned}
$$

Since

$\max _{\substack{i \neq 3 \\ i \neq 3}}\left\{w_{H} \frac{\partial y\left(x_{3}=0\right)}{\partial x_{i}}\right\}=w_{H} \frac{\partial y\left(x_{3}=0\right)}{\partial x_{4}}=4$

and

$\max _{\substack{i \neq 3 \\ i \neq 3}}\left\{w_{H} \frac{\partial y\left(x_{3}=1\right)}{\partial x_{i}}\right\}=w_{H} \frac{\partial y\left(x_{3}=1\right)}{\partial x_{1}}=5$

we write

$y=\overline{x_{3}}\left(\overline{x_{4}} y\left(x_{3}=0, x_{4}=0\right) \vee x_{4} y\left(x_{3}=0, x_{4}=1\right)\right)$

$\vee x_{3}\left(\overline{x_{1}} y\left(x_{1}=0, x_{3}=1\right) \vee x_{1} y\left(x_{1}=1, x_{3}=1\right)\right)$,

where

$y\left(x_{3}=0, x_{4}=0\right)=x_{5}, y\left(x_{3}=0, x_{4}=1\right)=\overline{x_{1}} \vee x_{2}$,
$y\left(x_{1}=0, x_{3}=1\right)=\overline{x_{2}} x_{5}$ and

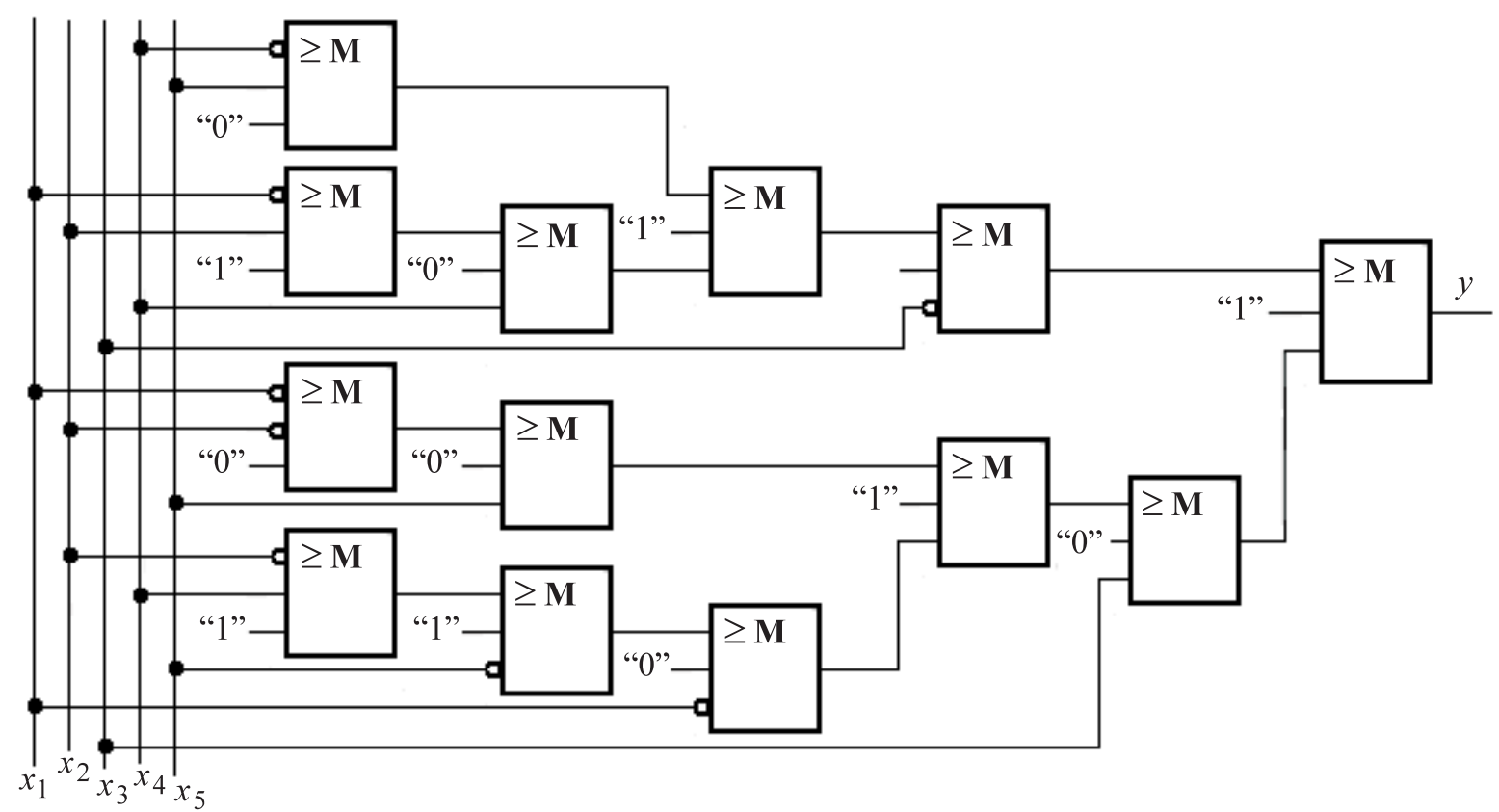

Fig. 7: Structural model with majority elements from Example 5 
$y\left(x_{1}=1, x_{3}=1\right)=\overline{x_{2}} \vee x_{4} \vee \overline{x_{5}}$, or

$y=\overline{x_{3}}\left(\overline{x_{4}} x_{5} \vee x_{4}\left(\overline{x_{1}} \vee x_{2}\right)\right) \vee x_{3}\left(\overline{x_{1}} \overline{x_{2}} x_{5} \vee x_{1}\left(\overline{x_{2}} \vee x_{4} \vee \overline{x_{5}}\right)\right)$.

In other words

$y=\left\{\overline{x_{3}} \#\left[\left(\overline{x_{4}} \# x_{5} \# 0\right) \#\left(x_{4} \#\left(\overline{x_{1}} \# x_{2} \# 1\right) \# 0\right) \# 1\right] \# 0\right\} \#$

$\#\left\{x_{3} \#\left[\left(\left(\overline{x_{1}} \# \overline{x_{2}} \# 0\right) \# x_{5} \# 0\right) \#\right.\right.$

$$
\left.\left.\#\left(x_{1} \#\left(\left(\overline{x_{2}} \# x_{4} \# 1\right) \overline{x_{5}} \# 1\right) \# 0\right) \# 1\right] \# 0\right\} \# 1 \text {. }
$$

and hence also the structural model (Fig. 7)

Obviously, there is also

$$
\begin{aligned}
x_{1} \# x_{2} \# x_{3} & =\left(x_{1} \vee x_{2}\right) x_{3} \vee x_{1} x_{2}=\left(x_{1} \oplus x_{2}\right) x_{3} \oplus x_{1} x_{2}= \\
& =S_{\{2,3\}}^{3}\left(x_{1}, x_{2}, x_{3}\right)=x_{1} x_{2}+x_{1} x_{3}+x_{2} x_{3}-2 x_{1} x_{2} x_{3} .
\end{aligned}
$$

\section{Conclusion}

It appears that it is feasible to produce symmetrical Boolean functions in a sufficiently simple way by a suitable control of one-digit binary adders or by numerical representation of values of the respective arithmetic polynomials, and to design logical circuits with majority elements by applying the Shannon decomposition of the given output function through effective selection of the arguments by which the decomposition is carried.

\section{References}

[1] Bochman, D., Posthoff, Ch.: Binare dynamische Systeme. Berlin: Akademie - Verlag, 1981.

[2] Harrison, M. A.: Introduction to Switching and Automata Theory. New York - Sydney: Mc Graw-Hill Book Co., 1965 .
[3] Frištacký, N. aj.: Logické systémy. Bratislava - Praha: ALFA - SNTL, 1986.

[4] Bokr, J., Jáneš, V.: "Some Interesting Applications of the Karnaugh Map.” Acta Electrotechnica et Informatica, Vol. 3 (2003), No. 3, p. 22-27.

[5] Bokr, J., Jáneš, V.: Logické systémy. Praha: Vydavatelství ČVUT, 1999.

[6] Šalyto, A. A.: Metody apparatnoj i programnoj relizacii algoritmov. Sankt - Peterburk: Nauka, 2000.

Doc. Ing. Josef Bokr, CSc. e-mail: bokr@kiv.zcu.cz

Department of Information and Computer Science

University of West Bohemia

Faculty of Applied Sciences

Universitní 22

30614 Pilsen, Czech Republic

Doc. Ing. Vlastimil Jáneš, CSc.

phone: +420224357289

e-mail: janes@fel.cvut.cz

Department of Computer Science and Engineering

Czech Technical University in Prague

Faculty of Electrical Engineering

Karlovo nám.13

12135 Praha 2, Czech Republic 